\title{
Enumeration of hypersurfaces with prescribed non-isolated singular subschemes
}

\author{
Weversson Dalmaso Sellin(1), Israel Vainsencher(i)
}

\begin{abstract}
Let $\mathbb{W}$ be an irreducible subvariety of a Hilbert scheme $\operatorname{Hilb}_{P_{W}(t)}\left(\mathbb{P}^{n}\right)$. We show under mild hypothesis that there are polynomial formulas for the degrees of the loci of hypersurfaces in $\mathbb{P}^{n}$ with singular subschemes containing some member of the family $\mathbb{W}$. The formulas are made explicit in a number of cases.
\end{abstract}

\section{Introduction}

The enumeration of singular hypersurfaces has a rich history. We refer the reader to Kleiman [20], [21] for a guide to the classical sources. Recent work has centered on generalizations of Göttsche's pioneering article [12]. Polynomial formulas have been shown to exist for the counting of any type of specified isolated singularities for hypersurfaces in higher dimensions, cf. Rennemo [27]; alas, his method is nonconstructive and doesn't lead to formulas. A different approach for the existence of universal polynomials

2010 AMS Subject Classification: 14N10 (Primary); 14C20, 14H20 (Secondary).

Key Words and Phrases: Enumerative Geometry, Bott's residues formula, singularities, Hilbert schemes.

This research was supported by UFVJM, UFMG and CNPq (grant 307495/2014-0). 
enumerating singular subvarieties is offered by Tzeng [31], via cobordism theory of bundles and divisors. A few special cases of explicit polynomial formulas, still for isolated singularities in higher dimensions, can be found in [32].

The purpose of this work is to investigate the loci of hypersurfaces with possibly nonisolated singularities. More precisely, given a closed, irreducible subvariety of a Hilbert scheme, $\mathbb{W} \subset \operatorname{Hilb}_{P_{W}(t)}\left(\mathbb{P}^{n}\right)$, we define a generalized discriminant subvariety $\Sigma(\mathbb{W}, d) \subset \mathbb{P}^{N_{d}}=\mathbb{P}\left(H^{0}\left(\mathcal{O}_{\mathbb{P}^{n}}(d)\right)\right)$, the points of which correspond to the hypersurfaces of degree $d$ in $\mathbb{P}^{n}$ singular along some (variable) member $W \in \mathbb{W}$. Assuming that a general member $W \in \mathbb{W}$ is smooth and of pure dimension $\leq n-2$, we show that the degree of $\Sigma(\mathbb{W}, d)$ is expressed by a polynomial $p^{\mathbb{W}}(d)$ for all $d \gg 0$. Our argument uses Grothendieck-Riemann-Roch. The degree of $p^{\mathbb{W}}(d)$ is shown to be bounded a priori by $n \operatorname{dim} \mathbb{W}$. The polynomial is made explicit for a few families $\mathbb{W}$, distinguished by an adequate description in the literature. Notably, we study the cases

$$
\begin{array}{ll}
\bullet \mathbb{W}_{(k, n)}:=\left\{\mathbb{P}^{k}-\text { linear in } \mathbb{P}^{n}\right\}, \\
\bullet \mathbb{W}_{m}:=\left\{\text { plane curves of degree } m \text { in } \mathbb{P}^{3}\right\}, \\
\bullet \mathbb{W}_{t w c}:=\left\{\text { twisted cubic curves in } \mathbb{P}^{3}\right\}, \\
\bullet \mathbb{W}_{r c}:=\left\{\text { ruled cubic surfaces in } \mathbb{P}^{4}\right\}, \\
\bullet \mathbb{W}_{\text {seg }}:=\left\{\text { Segre cubic } 3 \text {-folds in } \mathbb{P}^{5}\right\} \text { and } \\
\bullet \mathbb{W}_{\text {eqc }}:=\left\{\text { elliptic quartic curves in } \mathbb{P}^{3}\right\} .
\end{array}
$$

In all examples we actually find that the degree of our polynomial is

$$
\operatorname{deg} p^{\mathbb{W}}(d)=(k+1) \operatorname{dim} \mathbb{W}, \text { where } k=\operatorname{dim} W(\leq n-2), W \in \mathbb{W} \text {. }
$$

We conjecture this is always the case. Our main result is the following

Theorem 1. Notation and hypotheses as above, set $\mathcal{F}_{d}:=H^{0}\left(\mathbb{P}^{n}, \mathcal{O}_{\mathbb{P}^{n}}(d)\right)$. There exists a desingularization $\widetilde{\mathbb{W}} \rightarrow \mathbb{W}$ such that 
(i) for $d \gg 0$ there exists a vector subbunble $\mathcal{E}_{d} \subset \widetilde{\mathbb{W}} \times \mathcal{F}_{d}$ whose fiber over a general $W \in \widetilde{\mathbb{W}}$ is the subspace $H^{0}\left(\mathbb{P}^{n},\left(\mathcal{I}_{W}\right)^{2}(d)\right) \subset \mathcal{F}_{d}$ formed by homogeneous polynomials of degree $d$ with gradient null along $W$;

(ii) the map $\mathbb{P}\left(\mathcal{E}_{d}\right) \longrightarrow \mathbb{P}^{N_{d}}=\mathbb{P}\left(\mathcal{F}_{d}\right)$ induced by projection is generically injective and its image, $\Sigma(\mathbb{W}, d) \subset \mathbb{P}\left(\mathcal{F}_{d}\right)$, has degree

$$
\operatorname{deg} \Sigma(\mathbb{W}, d)=\int \operatorname{Segre}\left(w, \mathcal{E}_{d}\right) \cap[\widetilde{\mathbb{W}}],
$$

where $w=\operatorname{dim} \widetilde{\mathbb{W}}=\operatorname{dim} \mathbb{W}$.

(iii) $\operatorname{deg}(\Sigma(\mathbb{W}, d))$ is a polynomial in $d$ of degree $\leq n w$ for all $d \gg 0$.

Let us summarize the contents. $\S 2$ contains the proof of the theorem. The first step is to associate to a family $\mathbb{W}$ as above a family $\mathbb{W}^{\prime}$ of thickenings, cf. Def. 4. The general member $W^{\prime} \in \mathbb{W}^{\prime}$ has ideal $\mathcal{I}_{W^{\prime}}=$ $\left(\mathcal{I}_{W}\right)^{2}$ for $W \in \mathbb{W}$ a smooth member. A hypersurface of degree $d \gg 0$ is singular along $W$ if and only if its equation $F$ lies in $H^{0}\left(\mathbb{P}^{n}, \mathcal{I}_{W^{\prime}}(d)\right) \subset$ $\mathcal{F}_{d}$ (Lemma 5). The set of pairs $\left(W^{\prime}, F\right)$ such that $F \supset W^{\prime}$ is a vector subbundle $\mathcal{E}_{d}$ of $\mathbb{W}^{\prime} \times \mathcal{F}_{d}$. Our generalized discriminant $\Sigma(\mathbb{W}, d) \subset \mathbb{P}^{N_{d}}$ is the image of the projectivization $\mathbb{P}\left(\mathcal{E}_{d}\right)$, cf. (10). Standard techniques of intersection theory enable us to express $\operatorname{deg} \Sigma(\mathbb{W}, d)$ as a top Chern class of the quotient bundle $\mathcal{D}_{d}:=\mathcal{F}_{d} / \mathcal{E}_{d}$, cf. (12),(13). The latter bundle is a direct image, (7). Now GRR applies (14) to ensure that the desired top Chern class is a polynomial in $d$ of degree $\leq n \operatorname{dim} \mathbb{W}$.

Polynomial formulas for the families envisaged in (1) are derived via Bott's localization at fixed points (15), as we learn from Ellingsrud and Strømme [9] and Meurer [24]. The fixed points of $\mathbb{W}_{t w c}$ are available in op.cit. Additional work is required since our parameter space $\mathbb{W}_{t w c}^{\prime}$ is in fact a blowup of $\mathbb{W}_{t w c}$, cf. Prop. 14, Remark 15. Ditto for the families $\mathbb{W}_{\text {rc }}(\S 3.3 .2), \mathbb{W}_{\text {seg }}(\S 3.3 .3)$ and $\mathbb{W}_{\text {eqc }}(\S 3.4)$. We work over $\mathbb{C}$. 


\section{There are a vector bundle and a polynomial formula}

Let $\mathbb{W}$ be a closed, irreducible subvariety of a Hilbert scheme $\operatorname{Hilb}_{P_{\mathbb{W}}(t)}\left(\mathbb{P}^{n}\right)$. We assume the general member $W \in \mathbb{W}$ is smooth and of pure dimension $\leq n-2$. Let $W^{\prime} \subset \mathbb{P}^{n}$ be the subscheme with ideal sheaf $\mathcal{I}_{W^{\prime}}=\left(\mathcal{I}_{W}\right)^{2}$.

Lemma 2. Notation as just above, set $\mathcal{N}=\mathcal{I}_{W} /\left(\mathcal{I}_{W}\right)^{2}$. We have the formula for the Hilbert polynomials

$$
P_{W^{\prime}}(d):=\chi\left(\mathcal{O}_{W^{\prime}}(d)\right)=\chi\left(\mathcal{O}_{W}(d)\right)+\chi(\mathcal{N}(d)) .
$$

Proof. The assertion follows from the exact sequence

$$
0 \rightarrow \mathcal{N} \longrightarrow \mathcal{O}_{\mathbb{P}^{n}} /\left(\mathcal{I}_{W}\right)^{2}=\mathcal{O}_{W^{\prime}} \longrightarrow \mathcal{O}_{\mathbb{P}^{n}} / \mathcal{I}_{W}=\mathcal{O}_{W} \rightarrow 0 .
$$

Lemma 3. Notation as just above, we have a generically injective rational map

$$
\begin{aligned}
& \mathbb{W} \quad \rightarrow \operatorname{Hilb}_{P_{W^{\prime}}(t)}\left(\mathbb{P}^{n}\right) \\
& W \quad \mapsto \quad W^{\prime}, \mathcal{I}_{W^{\prime}}=\left(\mathcal{I}_{W}\right)^{2},
\end{aligned}
$$

which is a morphism on the open subset of $\mathbb{W}$ consisting of smooth members.

Proof. By Hirzebruch-Riemann-Roch [11, Cor.15.2.1,p. 288] and Conservation of Number [11, 10.21,p. 180], the r.h.s. in (3) is independent of the particular (smooth) $W$. By the universal property of Hilb [15], (see [8] for a wonderful introduction or [29] for the state of the art) the map exists over the open subset where flatness is ensured. Finally, if $Z, W \in \mathbb{W}$ are smooth members such that $\left(\mathcal{I}_{Z}\right)^{2}=\left(\mathcal{I}_{W}\right)^{2}$ it follows that $\mathcal{I}_{Z}=\mathcal{I}_{W}$.

Definition 4. Denote by $\mathbb{W}^{\prime}$ the closure of the image of the map (4).

Note the occurrence of a new Hilbert polynomial, $P_{\mathbb{W}^{\prime}}(t)$. For instance, if we take $\mathbb{W}$ as the family of lines in $\mathbb{P}^{3}$, we have $P_{\mathbb{W}}(t)=t+1$ whereas presently $P_{\mathbb{W}^{\prime}}(t)=3 t+1$. The latter is the Hilbert polynomial of the 
subscheme defined by the ideal $\left\langle x_{0}^{2}, x_{0} x_{1}, x_{1}^{2}\right\rangle=\left\langle x_{0}, x_{1}\right\rangle^{2}$. Our starting point is the elementary fact that a surface in $\mathbb{P}^{3}$ is singular along the line $\left\langle x_{0}, x_{1}\right\rangle$ if and only if its defining homogeneous polynomial lies in $\left\langle x_{0}, x_{1}\right\rangle^{2}$. Quite generally, to ask a hypersurface $F$ of degree $d$ to be singular along a general member $W \in \mathbb{W}$ is equivalent to requiring $F$ to be an element of $H^{0}\left(\left(\mathcal{I}_{W}\right)^{2}(d)\right)$. This approach was probably inaugurated by Harris and Pandharipande [17] and followed by Göttsche and Rennemo for isolated singularities.

We write $\operatorname{Sing}(F)$ for the singular locus of $F$. The next lemma is a main step towards the proof of Theorem.1(ii).

Lemma 5. Suppose $\mathcal{J}_{d}:=\left(\mathcal{I}_{W}\right)^{2}(d)$ globally generated. Let $F$ be a general element of $H^{0}\left(\mathcal{J}_{d}\right)$. Then $\operatorname{Sing}(F)=W$ set-theoretically.

Proof. The hypothesis that $\mathcal{J}_{d}$ be globally generated implies by Bertini (cf. $[18,10.9 .2]$ ) that $\operatorname{Sing}(F) \subseteq W$. The inclusion $W \subseteq \operatorname{Sing}(F)$ is evident: if $F$ lies in $H^{0}\left(\mathcal{J}_{d}\right)$ then its gradient is zero all along $W$.

Next we borrow from [1] the technical construction of the correspondence

$$
\widetilde{\Sigma}\left(\mathbb{W}^{\prime}, d\right):=\left\{(Z, F) \in \mathbb{W}^{\prime} \times \mathbb{P}^{N_{d}} \mid Z \subset F\right\} .
$$

Lemma 6. Notation as in Definition 4, consider the projection maps

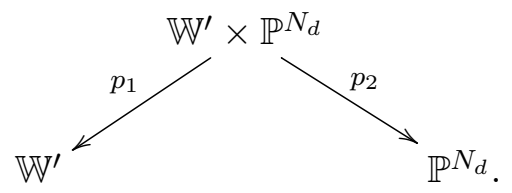

Then for all $d \gg 0$, the correspondence $\widetilde{\Sigma}\left(\mathbb{W}^{\prime}, d\right)$ (see $\left.(5)\right)$ is a projective bundle over $\mathbb{W}^{\prime}$ via the first projection $p_{1}$.

Proof. Let $\widetilde{Z} \subset \mathbb{W}^{\prime} \times \mathbb{P}^{n}$ be the universal subscheme and similarly $\widetilde{F} \subset$ $\mathbb{P}^{N_{d}} \times \mathbb{P}^{n}$ the universal hypersurface of degree $d$. Let us denote $\widehat{Z}, \widehat{F}$ their 
pullbacks to $\mathbb{W}^{\prime} \times \mathbb{P}^{N_{d}} \times \mathbb{P}^{n}$. We have the following diagram of sheaves

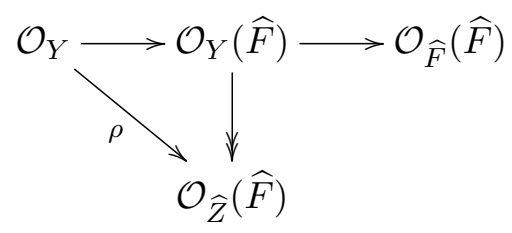

over $Y:=\mathbb{W}^{\prime} \times \mathbb{P}^{N_{d}} \times \mathbb{P}^{n}$. By construction, the oblique arrow $\rho$ vanishes at a point $(Z, F, x) \in Y$ if and only if $x \in F \cap Z$. So the inclusion $Z \subset F$ holds when the previous condition occurs for all $x \in Z$. Thus, $\widetilde{\Sigma}\left(\mathbb{W}^{\prime}, d\right)$ is equal to the scheme of zeros of $\rho$ along the fibers of the projection $p_{12}: \widehat{Z} \rightarrow \mathbb{W}^{\prime} \times \mathbb{P}^{N_{d}}$. Recalling Altman \& Kleiman [1, (2.1) p. 14], this is equal to the scheme of zeros of the adjoint section of the direct image vector bundle $p_{12 *}\left(\mathcal{O}_{\widehat{Z}}(\widehat{F})\right)$. Look at the projection maps

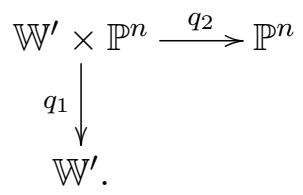

Since $\mathcal{O}(\widetilde{F})=\mathcal{O}_{\mathbb{P}^{N_{d}}}(1) \otimes \mathcal{O}_{\mathbb{P}^{n}}(d)$, by the projection formula we have produced a section of $\mathcal{O}_{\mathbb{P}^{N_{d}}}(1) \otimes \mathcal{D}_{d}$, where $\mathcal{D}_{d}=q_{1 *}\left(\mathcal{O}_{\widetilde{Z}}(d)\right)$. By CastelnuovoMumford and base change theory, there is an integer $d_{0}$ (= regularity) such that $\mathcal{D}_{d}$ is a vector bundle of rank $P_{\mathbb{W}^{\prime}}(d)$ for all $d \geq d_{0}$, where $P_{\mathbb{W}^{\prime}}(t)$ denotes the Hilbert polynomial of the members of $\mathbb{W}^{\prime}$. In fact, $\mathcal{D}_{d}$ fits into the exact sequence of vector bundles over $\mathbb{W}^{\prime}$ :

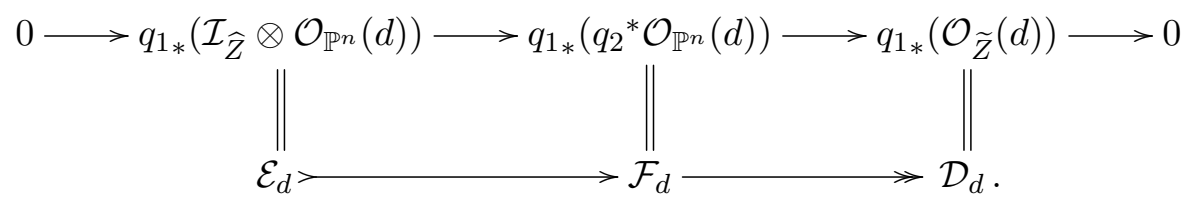

Taking the projectivization and pulling back to $\mathbb{W}^{\prime} \times \mathbb{P}^{N_{d}}$, we get (omitting pullbacks): 


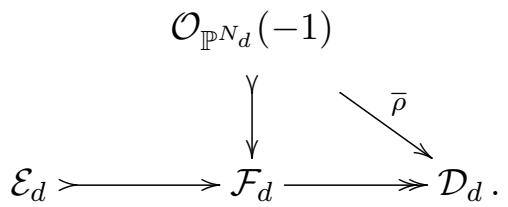

By construction, $\bar{\rho}$ vanishes precisely over $\widetilde{\Sigma}\left(\mathbb{W}^{\prime}, d\right)$. And this tells us that

$$
\widetilde{\Sigma}\left(\mathbb{W}^{\prime}, d\right)=\mathbb{P}\left(\mathcal{E}_{d}\right)
$$

Lemma 7. Notation as in $(7),(9)$ we have that $\widetilde{\Sigma}\left(\mathbb{W}^{\prime}, d\right)$ represents the top Chern class of $\mathcal{O}_{\mathbb{P}^{N_{d}}}(1) \otimes \mathcal{D}_{d}$.

Proof. As codim $\mathbb{W}_{\mathbb{W}^{\prime} \times \mathbb{P}^{N}} \tilde{\Sigma}\left(\mathbb{W}^{\prime}, d\right)=P_{\mathbb{W}^{\prime}}(d)$, which coincides with the rank of $\mathcal{D}_{d}$ due to $(7)$, the assertion follows from Fulton [11, 3.2.16, p. 61].

Definition 8. We call the $\mathbb{W}$-discriminant, denoted by $\Sigma(\mathbb{W}, d)$, the subvariety of $\mathbb{P}^{N_{d}}$ corresponding to the hypersurfaces which contain some member of $\mathbb{W}^{\prime}$.

For a general point $W^{\prime} \in \mathbb{W}^{\prime}$ and a hypersurface $F$, asking that $F \supset W^{\prime}$ as schemes is equivalent to requiring that the hypersurface be singular along the reduced scheme $W=W_{\text {red }}^{\prime} \in \mathbb{W}$. With the notation of $(6)$, we have

$$
\Sigma(\mathbb{W}, d)=p_{2}\left(\widetilde{\Sigma}\left(\mathbb{W}^{\prime}, d\right)\right)
$$

The usual discriminant hypersurface corresponds to the choice $\mathbb{W}=\mathbb{P}^{n}$.

Lemma 9. Notation as above, the map

$$
\begin{array}{rll}
\mathbb{W}^{\prime} \times \mathbb{P}^{N_{d}} \supset \widetilde{\Sigma}\left(\mathbb{W}^{\prime}, d\right) & \stackrel{p_{2}}{\longrightarrow} & \Sigma(\mathbb{W}, d) \subset \mathbb{P}^{N_{d}} \\
(Z, F) & \mapsto & F
\end{array}
$$

is generically injective for all $d>>0$. 
Proof. We must show that for a general $F \in \Sigma(\mathbb{W}, d)$, the fiber $p_{2}^{-1}(F) \subset$ $\widetilde{\Sigma}\left(\mathbb{W}^{\prime}, d\right)$ consists of a single element. In view of Lemma 3 , there is an open subset $\mathbb{W}_{0}^{\prime} \subset \mathbb{W}^{\prime}$ formed by subschemes $W^{\prime}$ with ideal of the form $\mathcal{I}_{W^{\prime}}=$ $\left(\mathcal{I}_{W}\right)^{2}$ with $W \in \mathbb{W}$ smooth. Now it suffices to show that the restriction of $p_{2}$ over $\mathbb{W}_{0}^{\prime}$ is injective. Let $F$ be a general hypersurface of degree $d$ containing $W^{\prime} \in \mathbb{W}_{0}^{\prime}$. This means that $F$ is general in $H^{0}\left(\mathcal{I}_{W^{\prime}}(d)\right)=$ $H^{0}\left(\left(\mathcal{I}_{W}\right)^{2}(d)\right)$. By Lemma 5 we have that $\operatorname{Sing}(F)=W$ (as sets). Let $Z^{\prime} \in \mathbb{W}_{0}^{\prime}$ be such that $Z^{\prime} \subset F$. So we have $\left(W^{\prime}, F\right)$ and $\left(Z^{\prime}, F\right) \in p_{2}^{-1}(F)$. By construction of $\mathbb{W}_{0}^{\prime}$, we have $\mathcal{I}_{Z^{\prime}}=\left(\mathcal{I}_{Z}\right)^{2}$ for some smooth $Z=Z_{\text {red }}^{\prime} \in$ $\mathbb{W}$. So $Z \subseteq \operatorname{Sing}(F)=W$. Since the Hilbert polynomials of $Z, W$ are one and the same, therefore $Z=W$ and so $Z^{\prime}=W^{\prime}$. This shows that the map in (11) is generically injective as asserted.

Lemma 10. Notation as above, we have

$$
\operatorname{deg} \Sigma(\mathbb{W}, d)=\int \operatorname{Segre}\left(w, \mathcal{E}_{d}\right) \cap\left[\mathbb{W}^{\prime}\right],
$$

where $w:=\operatorname{dim} \mathbb{W}^{\prime}=\operatorname{dim} \mathbb{W}$.

Proof. We have the equality of cycle classes

$$
\left(p_{2}\right)_{\star}\left[\widetilde{\Sigma}\left(\mathbb{W}^{\prime}, d\right)\right]=[\Sigma(\mathbb{W}, d)] .
$$

This follows from $[11, \S 1.4$, p. 11$]$ since $\widetilde{\Sigma}(\mathbb{W}, d) \stackrel{p_{2}}{\longrightarrow} \Sigma(\mathbb{W}, d)$ is birational, as shown in Lemma 9. Set $\delta:=\operatorname{dim} \Sigma(\mathbb{W}, d)$. We have $\delta=w+\epsilon$, with $\epsilon:=\operatorname{rk} \mathcal{E}_{d}-1$. Set $H=c_{1} \mathcal{O}_{\mathbb{P}^{N}}(1)$, the hyperplane class. By projection formula we may write

$$
\begin{aligned}
\operatorname{deg} \Sigma(\mathbb{W}, d) & =\int H^{\delta} \cap[\Sigma(\mathbb{W}, d)]=\int p_{2}^{\star} H^{\delta} \cap\left[\widetilde{\Sigma}\left(\mathbb{W}^{\prime}, d\right)\right] \\
& =\int\left(p_{1}\right)_{\star}\left(p_{2}^{\star} H^{w+\epsilon} \cap\left[\widetilde{\Sigma}\left(\mathbb{W}^{\prime}, d\right)\right]\right)=\int \operatorname{Segre}\left(w, \mathcal{E}_{d}\right) \cap\left[\mathbb{W}^{\prime}\right],
\end{aligned}
$$

using Fulton [11, §3.1, p. 47, Prop.4.4,p. 83 and Ex. 8.3.14, p. 143].

Proposition 11. The degree of the $\mathbb{W}$-discriminant, $\Sigma(\mathbb{W}, d)$, is a polynomial in $d$ of degree $\leq n \operatorname{dim}(\mathbb{W})$ for all $d \gg 0$. 
Proof. Let $\widetilde{\mathbb{W}} \rightarrow \mathbb{W}^{\prime}$ be a desingularization (cf. [19]). Pulling back $\mathcal{E}_{d}, \mathcal{D}_{d}$ in (7) to $\widetilde{\mathbb{W}}$, we may as well simplify notation and assume $\widetilde{\mathbb{W}}=\mathbb{W}^{\prime}$ smooth. We now argue as in [6] and [33]. Recall $\mathcal{D}_{d}$ is a direct image of a sheaf over $\mathbb{W}^{\prime} \times \mathbb{P}^{n}$ (cf. 7$)$. The same diagram of sheaves tells us

$$
\operatorname{Segre}\left(w, \mathcal{E}_{d}\right)=c_{w}\left(\mathcal{D}_{d}\right) .
$$

Now we can apply Grothendieck-Riemann-Roch (cf. [11, Thm.15.2, p. 286]) to express the Chern character of $\mathcal{D}_{d}$ as

$$
\operatorname{ch}\left(\mathcal{D}_{d}\right)=\operatorname{ch}\left(\left(q_{1}\right)_{!}\left(\mathcal{O}_{\widetilde{Z}}(d)\right)\right)=\left(q_{1}\right)_{*}\left(\operatorname{ch}\left(\mathcal{O}_{\widetilde{Z}}\right) \cdot \operatorname{ch}\left(\mathcal{O}_{\mathbb{P}^{n}}(d)\right) \cdot \operatorname{todd}\left(\mathbb{P}^{n}\right)\right) .
$$

Note that the right hand side is a polynomial in $d$ of degree $\leq n$. On the other hand, the Chern class $c_{w}$ is a weighted polynomial of degree $w$ on the coefficients of the Chern character $([11,3.2 .3$, p. 56]). This implies that $c_{w}\left(\mathcal{D}_{d}\right)$ is a polynomial in $d$ of degree $\leq n w$.

Remark 12. In order to get a polynomial formula, it suffices to calculate the degree of $\Sigma(\mathbb{W}, d)$ for $n \operatorname{dim} \mathbb{W}+1$ values of $d$. In all cases treated in this work, we find that the degree of the polynomial $p^{\mathbb{W}}(d)$ actually is $(k+1) \times \operatorname{dim}(\mathbb{W})$, where $k$ denotes the dimension of a member of $\mathbb{W}$. The validity for $\mathbb{W}$ arbitrary remains conjectural. [31] and [27] handle the case $k=0$.

To compute explicitly the integral in (12), we will apply Bott's residues formula in the equivariant flavor of [9] (see also [23], [24]),

$$
\int \operatorname{Segre}\left(w, \mathcal{E}_{d}\right) \cap\left[\mathbb{W}^{\prime}\right]=\sum_{F} \frac{c_{w}^{\mathbb{T}}\left(-\mathcal{E}_{d}\right) \cap[F]_{\mathbb{T}}}{c_{\text {top }}^{\mathbb{T}}\left(\mathcal{N}_{F \mid \mathbb{W}^{\prime}}\right)},
$$

where the sum runs through all fixed components $F$ of a convenient action of the torus $\mathbb{T}:=\mathbb{C}^{*}$ on $\mathbb{W}^{\prime}$. The $\mathcal{N}_{F \mid \mathbb{W}^{\prime}}$ appearing in the denominator denotes the normal bundle of a fixed component $F$ in $\mathbb{W}^{\prime}$. In all cases treated in this work the set of fixed points is finite. Thus the denominator in (15) is the $\mathbb{T}$-equivariant top Chern class, $c_{\text {top }}^{\mathbb{T}}\left(\mathcal{T}_{F} \mathbb{W}^{\prime}\right)$, where $\mathcal{T}_{F} \mathbb{W}^{\prime}$ denotes the tangent space at a fixed point $F$ in $\mathbb{W}^{\prime}$. 
Remark 13. Notation as in (1), for $\mathbb{W}=\mathbb{W}_{(k, n)}$ (as well as $\mathbb{W}=\mathbb{W}_{m}$ ), the family $\mathbb{W}^{\prime}$ which parameterizes subschemes of $\mathbb{P}^{n}$ defined by $\left(I_{W}\right)^{2}$ with $W \in \mathbb{W}$ is flat. In fact, we have $\mathbb{W}=\mathbb{W}^{\prime}$ : the map (4) is an isomorphism. However, in the other cases dealt with in this work we have only the generic flatness guaranteed over the locus of smooth $W \in \mathbb{W}$. In fact, the Hilbert polynomial for $\left(I_{W}\right)^{2}$ may jump at special points. A blowup will be required in order to achieve flatness, following Raynaud [26].

\section{Enumerative results}

"For many problems it would be miraculous and totally unexpected if somebody were to find a precise formula for the solution; most of the time one must settle for a rough estimate instead." ${ }^{1}$

A detailed exposition of the fixed points and the computations of their contributions on Bott's formula (15), including scripts for Macaulay2 [14], Maple [22] and Singular [7] and for the resolution of indeterminacies in the cases of sections 3.3 and 3.4 can be found in Sellin [28].

\subsection{Hypersurfaces singular along a linear $\mathbb{P}^{k} \subset \mathbb{P}^{n}$}

Here, the parameter space $\mathbb{W}_{(k, n)}:=\mathbb{G}(k+1, n+1)$, the grassmannian of $k+1$ dimensional vector subspaces of $\mathbb{C}^{n+1}$. Our goal is to determine the degree of the family of hypersurfaces of degree $d$ singular along some $\mathbb{P}^{k} \subset \mathbb{P}^{n}$.

For the reader's benefit we will show the calculations for $\operatorname{deg} \Sigma\left(\mathbb{W}_{(1,3)}, d\right)$.

Consider the torus $\mathbb{T}=\mathbb{C}^{*}$ acting diagonally on $\mathcal{F}_{1}=\left(\mathbb{C}^{4}\right)^{\vee}$ via

$$
t \circ x_{i}:=t^{w_{i}} x_{i}
$$

with appropriate weights, say:

$$
w_{0}=4, w_{1}=11, w_{2}=17, w_{3}=32 ;
$$

The requirement is that denominators appearing in (15), which turn out to be polynomials in the weights $w_{0}, \ldots, w_{3}$, do not vanish.

\footnotetext{
${ }^{1}$ Tim Gowers, Mathematics: A Very Short Introduction
} 
We get a natural induced action on $\mathbb{W}_{(1,3)}=\mathbb{G}(2,4)$. The tautological vector bundles

$$
\mathcal{S} \longrightarrow \mathcal{F}_{1} \longrightarrow \mathcal{Q}
$$

on $\mathbb{W}_{(1,3)}$ are $\mathbb{T}$-equivariant. The fiber of $\mathcal{S}$ over a line $l \in \mathbb{G}(2,4)$ is the two dimensional subspace of $\mathcal{F}_{1}$ of linear forms vanishing on $l$. Presently we have six fixed points corresponding to the coordinate axes

$$
\left\langle x_{0}, x_{1}\right\rangle,\left\langle x_{0}, x_{2}\right\rangle, \ldots,\left\langle x_{2}, x_{3}\right\rangle \text {. }
$$

Referring to (15), we have

$$
\operatorname{deg} \Sigma\left(\mathbb{W}_{(1,3)}, d\right)=\sum_{F} \frac{c_{4}^{\mathbb{T}}\left(-\mathcal{E}_{d}\right) \cap[F]_{\mathbb{T}}}{c_{4}^{\mathbb{T}}\left(\mathcal{T} \mathbb{W}_{(1,3)}\right)}
$$

summing over the six fixed points. The denominator in (17), let's say for $F=\left\langle x_{0}, x_{1}\right\rangle$, is obtained as follows. First we find the fiber of the tangent

$$
\mathcal{T}_{F} \mathbb{W}_{(1,3)}=\operatorname{Hom}\left(\mathcal{S}_{F}, \mathcal{Q}_{F}\right)=\left\langle x_{0}, x_{1}\right\rangle^{\vee} \otimes\left\langle x_{2}, x_{3}\right\rangle=\frac{x_{2}}{x_{0}}+\frac{x_{3}}{x_{0}}+\frac{x_{2}}{x_{1}}+\frac{x_{3}}{x_{1}},
$$

where $\frac{x_{i}}{x_{j}}$ denotes the $\mathbb{T}$-space with weight $w_{i}-w_{j}$. In this way, we obtain $c_{4}^{\mathbb{T}}\left(\mathcal{T} \mathbb{W}_{(1,3)}\right) \cap[F]_{\mathbb{T}}=\left(w_{2}-w_{0}\right)\left(w_{3}-w_{0}\right)\left(w_{2}-w_{1}\right)\left(w_{3}-w_{1}\right)$. With the choice of weights in (16), this gives us the value 45864. Similarly, the numerator requires the weight decomposition of the fiber $\left(\mathcal{E}_{d}\right)_{F}$. To fix the ideas, take $d=3$. Now that fiber consists of the cubic forms $f \in H^{0}\left(\mathcal{O}_{\mathbb{P}^{3}}(3)\right)$ with gradient null along the line $F$. The weight decomposition is given by

$\left(\mathcal{E}_{3}\right)_{F}=x_{0}^{3}+x_{0}^{2} x_{1}+x_{0}^{2} x_{2}+x_{0}^{2} x_{3}+x_{0} x_{1}^{2}+x_{0} x_{1} x_{2}+x_{0} x_{1} x_{3}+x_{1}^{3}+x_{1}^{2} x_{2}+x_{1}^{2} x_{3}$.

Since we actually need the Segre class, $\operatorname{Chern}\left(-\mathcal{E}_{d}\right)=\operatorname{Chern}\left(\mathcal{D}_{d}\right)$ cf. $(7)$, we find the complementary decomposition

$\left(\mathcal{D}_{d}\right)_{F}=x_{0} x_{2}^{2}+x_{1} x_{2}^{2}+x_{2}^{3}+x_{0} x_{2} x_{3}+x_{1} x_{2} x_{3}+x_{2}^{2} x_{3}+x_{0} x_{3}^{2}+x_{1} x_{3}^{2}+x_{2} x_{3}^{2}+x_{3}^{3}$.

Here $x_{i}^{\alpha} x_{j}^{\beta} x_{k}^{\gamma}$ denotes the $\mathbb{T}$-space with weight $\alpha w_{i}+\beta w_{j}+\gamma w_{k}$. The corresponding numerical contribution is 3217978137 . The fixed point 
$F=\left\langle x_{0}, x_{1}\right\rangle$ contributes the fraction $3217978137 / 45864$. The total contribution of the six fixed points is $\frac{3217978137}{45864}-\frac{2152229961}{17640}+\frac{774359841}{28665}+\frac{1227942219}{28665}-\frac{392711889}{17640}+\frac{218302833}{45864}=504$.

This is the degree of the subvariety of $\left|\mathcal{O}_{\mathbb{P}}^{3}(3)\right|=\mathbb{P}^{19}$ consisting of the Whitney umbrellas: surfaces of degree 3 in $\mathbb{P}^{3}$ which are singular along some line (cf. [5]).

Recalling Remark 12, we need the degrees of $\Sigma\left(\mathbb{W}_{(1,3)}, d\right)$ for $3 \times 4+1$ values of $d$. Interpolating, we get

$\operatorname{deg} \Sigma\left(\mathbb{W}_{(1,3)}, d\right)=\frac{1}{32}\left(\begin{array}{l}d \\ 2\end{array}\right)\left(27 d^{6}-117 d^{5}+269 d^{4}-375 d^{3}+312 d^{2}-132 d+48\right)$.

We list below the results for $(k, n) \in\{(2,4),(2,5),(3,5)\}$ :

$$
\begin{gathered}
\operatorname{deg} \Sigma\left(\mathbb{W}_{(2,4)}, d\right)=\frac{1}{2^{7} \cdot 3^{3}}\left(\begin{array}{c}
d+2 \\
4
\end{array}\right)\left(9 d^{14}-18 d^{13}-63 d^{12}\right. \\
+396 d^{11}-405 d^{10}-1530 d^{9}+5328 d^{8}-4176 d^{7}-9414 d^{6} \\
\left.+27208 d^{5}-24347 d^{4}-4696 d^{3}+36572 d^{2}-32544 d+14400\right) . \\
\operatorname{deg} \Sigma\left(\mathbb{W}_{(2,5)}, d\right)=\frac{1}{(2)^{3} 3^{11} 5^{3}}\left(\begin{array}{c}
d+2 \\
4
\end{array}\right)\left(12800 d^{23}-25600 d^{22}\right. \\
-224000 d^{21}+966400 d^{20}+520800 d^{19} \\
+10632000 d^{18}+18128000 d^{17}+35186000 d^{16}-170677265 d^{15} \\
+2164141556 d^{10}-5208921230 d^{9}+3728975455 d^{8}+3332483181 d^{7} \\
-10452711042 d^{6}+10781927010 d^{5}-2523245175 d^{4}-7609562253 d^{3} \\
\left.+11511503406 d^{2}-8323547040 d+3637418400\right) . \\
\operatorname{deg} \Sigma\left(\mathbb{W}_{(3,5)}, d\right)=\frac{1}{2^{27} \cdot 3^{3} \cdot 5^{4}}\left(\begin{array}{c}
d+2 \\
4
\end{array}\right)\left(1125 d^{28}+15750 d^{27}\right. \\
+86625 d^{26}+168750 d^{25}-187875 d^{24}-38250 d^{23} \\
+8824725 d^{22}+23473350 d^{21}-32467725 d^{20}-128183670 d^{19}
\end{gathered}
$$




$$
\begin{gathered}
+426415635 d^{18}+1377078570 d^{17}-2137554049 d^{16} \\
-7117020302 d^{15}+15925316455 d^{14}+37514746370 d^{13} \\
-82840806388 d^{12}-125157483544 d^{11}+422227932240 d^{10} \\
+287672117600 d^{9}-1529648949952 d^{8}+207120164224 d^{7} \\
+4517312266240 d^{6}-3047085731840 d^{5}-6253154779136 d^{4} \\
+11893749153792 d^{3}+2911913902080 d^{2} \\
-8455245004800 d+2378170368000) .
\end{gathered}
$$

Recalling $\operatorname{dim} \mathbb{W}_{k, n}=(k+1)(n-k)$, we remark that the degrees of the above polynomials are in agreement with the expectation (2), to wit, $(k+1) \operatorname{dim} \mathbb{W}$.

\subsection{Surfaces singular along plane curves}

The family of plane curves of degree $m>1$ in $\mathbb{P}^{3}$ is parameterized by a $\mathbb{P}^{N_{m}}$-bundle over $\check{\mathbb{P}}^{3}$

$$
\mathbb{W}_{m} \longrightarrow \check{\mathbb{P}}^{3},
$$

where $N_{m}=\left(\begin{array}{c}m+2 \\ 2\end{array}\right)-1$. We have calculated $\operatorname{deg} \Sigma\left(\mathbb{W}_{m}, d\right)$ for $m=2,3$ :

$$
\begin{gathered}
\operatorname{deg} \Sigma\left(\mathbb{W}_{2}, d\right)=\frac{1}{2^{13} \cdot 3^{2} \cdot 5 \cdot 7}(d-2)\left(150903 d^{15}-3809754 d^{14}\right. \\
+44834472 d^{13}-317080224 d^{12}+1422290970 d^{11}-3579080844 d^{10} \\
-455933988 d^{9}+47928493544 d^{8}-237841700217 d^{7}+712127741206 d^{6} \\
-1498533401372 d^{5}+2287674925704 d^{4}-2504345972608 d^{3} \\
\left.+1873638158208 d^{2}-859900216320 d+182801203200\right) .
\end{gathered}
$$




$$
\begin{gathered}
\operatorname{deg} \Sigma\left(\mathbb{W}_{3}, d\right)=\frac{1}{2^{20} \cdot 3^{4} \cdot 5 \cdot 7 \cdot 11}\left(13286025 d^{24}-1038081420 d^{23}\right. \\
+39146062158 d^{22}-946074434976 d^{21}+16407919974303 d^{20} \\
\quad-216603408547548 d^{19}+2251372103607528 d^{18} \\
-18776305509313968 d^{17}+126579622223230407 d^{16} \\
-686155959955971780 d^{15}+29119998634468665566 d^{14} \\
-8886007643094113376 d^{13}+12799827743693355329 d^{12} \\
+50456388588134712812 d^{11}-483658040042985949724 d^{10} \\
+2229927488252098274992 d^{9}-7358275057877141245584 d^{8} \\
+18804143410678335462720 d^{7}-38007885859704936084800 d^{6} \\
+60658830486712279959808 d^{5}-75133955486596446561280 d^{4}+ \\
69793667761693681135616 d^{3}-45744106516543857328128 d^{2} \\
+18819557445986636267520 d-3636764182567924531200) .
\end{gathered}
$$

The reader interested in obtaining $\operatorname{deg} \Sigma\left(\mathbb{W}_{m}, d\right)$ for other $m$, simply plug in the desired value in the script in [28, Appendix E, p.92]. Notice the degrees of the above polynomials in $(22)$ and $(23)$ are $(k+1) \operatorname{dim} \mathbb{W}=$ $2\left(2+\left(\begin{array}{c}d+2 \\ 2\end{array}\right)\right)$.

\subsection{Hypersurfaces singular along base loci of nets of quadrics of determinantal type}

In this section we discuss the case of hypersurfaces in $\mathbb{P}^{n}(n=3,4,5)$ singular along base loci of nets of quadrics of determinantal type. By this we mean the nets generated by $2 \times 2$-minors of a $3 \times 2$ matrix of linear forms. Specifically, we consider the families

$$
\left\{\begin{array}{l}
\mathbb{W}_{t w c}=\left\{\text { twisted cubics in } \mathbb{P}^{3}\right\}, \\
\mathbb{W}_{r c}=\left\{\text { ruled cubics in } \mathbb{P}^{4}\right\} \text { and } \\
\mathbb{W}_{\text {seg }}=\left\{\text { Segre } 3 \text {-folds in } \mathbb{P}^{5}\right\} .
\end{array}\right.
$$




\subsubsection{Surfaces singular along twisted cubics}

A twisted cubic is a rational, smooth curve of degree 3 in $\mathbb{P}^{3}$. Any such is projectively equivalent to the scheme of zeros of the $2 \times 2$ minors of the matrix $\left(\begin{array}{lll}x_{0} & x_{1} & x_{2} \\ x_{1} & x_{2} & x_{3}\end{array}\right)$. Its Hilbert polynomial is $3 t+1$. Piene \& Schlessinger $[25]$ showed that the component $\mathbb{W}_{t w c} \subset \operatorname{Hilb}_{3 t+1}\left(\mathbb{P}^{3}\right)$ is a smooth projective variety of dimension 12. Subsequently, Ellingsrud, Piene \& Strømme [10] proved that the subvariety of the Grassmannian

$$
\mathbb{X} \subset \mathbb{G}\left(3, \mathcal{F}_{2}\right)
$$

formed by nets of determinantal type is smooth. Moreover the component $\mathbb{W}_{t w c}$ is the blowup of $\mathbb{X}$ along the subvariety $\mathbb{G}_{\omega}$ of nets projectively equivalent to the net

$$
\omega:=\left(x_{0}^{2}, x_{0} x_{1}, x_{0} x_{2}\right) .
$$

A typical element on the fiber of the exceptional divisor over $\omega$ corresponds to an ideal of the form $\mathcal{I}_{\omega, f}:=\left\langle x_{0}^{2}, x_{0} x_{1}, x_{0} x_{2}, f\right\rangle$, where $x_{0}=$ $f\left(x_{1}, x_{2}, x_{3}\right)=0$ is a plane cubic singular at the point $x_{0}=x_{1}=x_{2}=$ 0 . The square $\left(\mathcal{I}_{\omega, f}\right)^{2}$ of any such ideal has Hilbert polynomial $9 t-$ 7 , same as for the square of the ideal of the standard twisted cubic, $\left\langle x_{1} x_{3}-x_{2}^{2}, x_{0} x_{3}-x_{1} x_{2}, x_{1}^{2}-x_{0} x_{2}\right\rangle$.

Unlike the cases $\mathbb{W}_{(k, n)}$ and $\mathbb{W}_{m}$, the family formed by the subschemes of $\mathbb{P}^{3}$ defined by $\left(\mathcal{I}_{W}\right)^{2}, W \in \mathbb{W}_{t w c}$ is not flat. In fact, the element

$$
\mathbf{o}:=\left\langle x_{0}, x_{1}\right\rangle^{2}=\left\langle x_{0}^{2}, x_{0} x_{1}, x_{1}^{2}\right\rangle
$$

is a member of the good component $\mathbb{W}_{t w c}$, but its square has "bad" Hilbert polynomial, namely $P_{\mathbb{W}_{t w c}}(t)=10 t-10$, instead of $9 t-7$.

This is remedied by blowing up $\mathbb{W}_{t w c}$ along the orbit $\mathbb{G}_{\mathbf{0}}$. Since $\mathbb{G}_{\mathbf{0}} \cap$ $\mathbb{G}_{\omega}=\emptyset$, it follows that $\mathbb{G}_{\mathbf{o}}$ lifts isomorphically to an orbit in $\mathbb{W}_{t w c}$, still denoted by $\mathbb{G}_{\mathbf{0}}$. Let $\mathbb{W}_{t w c}^{\prime}$ denote the blowup of $\mathbb{W}_{t w c}$ along $\mathbb{G}_{\mathbf{0}}$. In fact, $\mathbb{X}$ and $\mathbb{W}_{t w c}$ are isomorphic over any neighborhood of $\mathbb{G}_{\mathbf{o}}$ disjoint from $\mathbb{G}_{\omega}$. The restriction $\mathbb{W}_{t w c \mid \mathbb{X} \backslash \mathbb{G}_{\omega}}^{\prime}$ is isomorphic to the restriction $\mathbb{X}_{\mid \mathbb{X} \backslash \mathbb{G}_{\omega}}^{\prime}$ of the blowup $\mathbb{X}^{\prime}$ of $\mathbb{X}$ along $\mathbb{G}_{\mathbf{0}}$. 
Let $\mathcal{C}$ be the tautological subbundle of rank 3 over the grassmannian of nets of quadrics $\mathbb{G}\left(3, \mathcal{F}_{2}\right)$. Write $\mathrm{S}_{2}(\mathcal{C})$ the symmetric power.

Proposition 14. Let $\mu: \mathrm{S}_{2}(\mathcal{C})_{\mid \mathbb{W}_{t w c}} \rightarrow \mathcal{F}_{4}$ be the natural map induced by multiplication. Consider the blowing up diagram of $\mathbb{W}_{t w c}$ along $\mathbb{G}_{\mathbf{o}}$

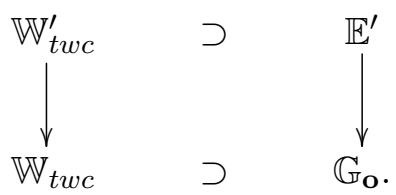

Then (i) $\mathbb{G}_{\mathbf{o}}$ is the scheme of zeros of $\wedge^{\wedge} \mu$;

(ii) $\mathbb{W}_{t w c}^{\prime}$ embeds in $\mathbb{W}_{t w c} \times \mathbb{G}\left(6, \mathcal{F}_{4}\right)$ as the closure of the graph of the rational map $\mathbb{W}_{t w c^{-\rightarrow} \rightarrow \mathbb{G}}\left(6, \mathcal{F}_{4}\right)$ induced by $\mu$.

(iii) The fiber of the exceptional divisor $\mathbb{E}^{\prime}$ over $\mathbf{o}$ is the projectivization of the quotient space of quartic forms,

$$
\left(\left\langle x_{0}, x_{1}\right\rangle^{3}\right)_{4} /\left(\left\langle x_{0}, x_{1}\right\rangle^{4}\right)_{4}
$$

Proof. The argument is based on local calculations as shown in [28, Appendix F.1]. We just highlight the main steps. Denote by $\mathfrak{Z}$ the scheme of zeros in question; it is invariant under the natural $\mathbb{P} \mathbb{G L}_{4}$ induced action. Recall $\mathbb{X}(24)$ has precisely two closed orbits, represented by the nets

$$
\mathbf{o}=\left(x_{0}^{2}, x_{0} x_{1}, x_{1}^{2}\right) \text { and } \omega=\left(x_{0}^{2}, x_{0} x_{1}, x_{0} x_{2}\right) .
$$

Clearly $\mathbf{o} \in \mathfrak{Z} \not \supset \omega$. Consider the list the 10 quadratic monomials,

$$
m_{1}:=x_{0}^{2}, m_{2}:=x_{0} x_{1}, m_{3}:=x_{1}^{2}, m_{4}:=x_{0} x_{2}, \ldots, m_{10}:=x_{3}^{2} .
$$

Use the affine coordinates $a_{i j}, 1 \leq i \leq 3,1 \leq j \leq 7$ for the open subset $\mathbb{G}^{0} \subset \mathbb{G}(3,10)$ so that the quadrics

$$
\left\{\begin{array}{rlrlrl}
q_{1}:=x_{0}^{2} & & & +\sum a_{1 j} m_{3+j} \\
q_{2}:= & & x_{0} x_{1} & & +\sum a_{2 j} m_{3+j} \\
q_{3}:= & & & x_{1}^{2} & +\sum a_{3 j} m_{3+j}
\end{array}\right.
$$

yield a trivialization for the restriction $\mathcal{C}_{\mid \mathbb{G}^{0}}$. Over $\mathbb{G}^{0}$ the multiplication map $\mathcal{C} \otimes \mathcal{F}_{1} \rightarrow \mathcal{F}_{3}$ is of generic rank 12 ; the rank drops to 10 exactly along $\mathbb{X}^{0}:=\mathbb{X} \cap \mathbb{G}^{0}=\mathbb{W}_{t w c} \cap \mathbb{G}^{0}=: \mathbb{W}_{t w c}^{0}$; the 2 nd equality stems from 
the fact that $\mathbb{G}^{0}$ is a neighborhood away from the orbit of $\omega$. This yields explicit equations for $\mathbb{X}^{0} \subset \mathbb{G}^{0}$. These equations allow us to express 9 of the coordinates in terms of the 12 remaining ones; these in turn provide affine coordinates for $\mathbb{X}^{0}$. Working out a matrix representation for $\mu: \mathcal{S}_{2} \mathcal{C}_{\mid \mathbb{X}^{0}} \rightarrow \mathcal{F}_{4}$ we find that the ideal of $6 \times 6$ minors, which defines $\mathfrak{Z}$, is equal to the ideal of $\left(\mathbb{G}_{\mathbf{o}}\right)^{0}:=\mathbb{G}_{\mathbf{0}} \cap \mathbb{X}^{0} \subset \mathbb{X}^{0}$. Since $\mathfrak{Z} \supseteq \mathbb{G}_{\mathbf{0}}$ are closed invariant subschemes which agree in a neighborhood of their unique closed orbit, they must be equal. Blowing it up, we get $\mathbb{X}^{\prime}$ (resp. $\mathbb{W}_{t w c}^{\prime}$ ) embedded in $\mathbb{X} \times \mathbb{G}\left(6, \mathcal{F}_{4}\right)$ (resp. $\mathbb{W}_{t w c} \times \mathbb{G}\left(6, \mathcal{F}_{4}\right)$ as the closure of the graph of the rational map induced by $\mu$. Likewise, we find that the fiber of $\mathbb{E}^{\prime}$ over $\mathbf{o}$ is as stated in (iii).

Remark 15. The previous result implies that the fixed points in $\mathbb{W}_{t w c}^{\prime}$ are obtained from those well known for $\mathbb{W}_{t w c}$ (cf. [9]), except for the six ones belonging to $\mathbb{G}_{\mathbf{0}}$. For each of these, say $\mathbf{o}=\left\langle x_{0}, x_{1}\right\rangle^{2}$, we form the ideals $\left\langle x_{0}, x_{1}\right\rangle^{4}+\langle Q\rangle, Q \in\left\{x_{0}^{3} x_{2}, x_{0}^{3} x_{3}, x_{0}^{2} x_{1} x_{2}, x_{0}^{2} x_{1} x_{3}, x_{0} x_{1}^{2} x_{2}, x_{0} x_{1}^{2} x_{3}, x_{1}^{3} x_{2}, x_{1}^{3} x_{3}\right\}$. These eight monomials span the exceptional fiber $\left(\left\langle x_{0}, x_{1}\right\rangle^{3}\right)_{4} /\left(\left\langle x_{0}, x_{1}\right\rangle^{4}\right)_{4}$.

For details about the explicit contribution of each fixed point the reader is again kindly referred to [28, Appendix F.3]. The polynomial that gives us the degree of $\Sigma\left(\mathbb{W}_{t w c}, d\right)$ is displayed in (27). Note that its degree is equal to $2 \times \operatorname{dim}\left(\mathbb{W}_{t w c}\right)=2 \times 12$ in agreement with $(2)$.

$$
\begin{gathered}
\operatorname{deg} \Sigma\left(\mathbb{W}_{t w c}, d\right)=\frac{1095687}{50462720} d^{24}-\frac{19230291}{18022400} d^{23}+\frac{24114591}{985600} d^{22} \\
-\frac{3932462817}{11468800} d^{21}+\frac{73665592101}{22937600} d^{20}-\frac{23321377833}{1146880} d^{19}+\frac{4087404048523}{51609600} d^{18} \\
-\frac{205245946577}{2457600} d^{17}-\frac{79029321809671}{68812800} d^{16}+\frac{2854774357217311}{309657600} d^{15} \\
-\frac{6688891988137}{143360} d^{14}+\frac{895445339622112187}{3406233600} d^{13}-\frac{4177328126526143027}{2270822400} d^{12} \\
+\frac{1134029525022301939}{94617600} d^{11}-\frac{29052565860084958379}{464486400} d^{10}+\frac{1100107099486708819}{4300800} d^{9} \\
-\frac{31950097995158831119}{38707200} d^{8}+\frac{365421773568911927}{172800} d^{7}-\frac{8318629615873057099}{1935360} d^{6} \\
+\frac{615395937691427021}{89600} d^{5}-\frac{337777058982513508747}{39916800} d^{4}+\frac{5167781409451915223}{665280} d^{3} \\
-\frac{693707469384158233}{138600} d^{2}+\frac{466431399017887}{231} d-383398629664 .
\end{gathered}
$$


Figure 1 shows with the help of Surfer [30] an example of a quartic surface singular along a twisted cubic.

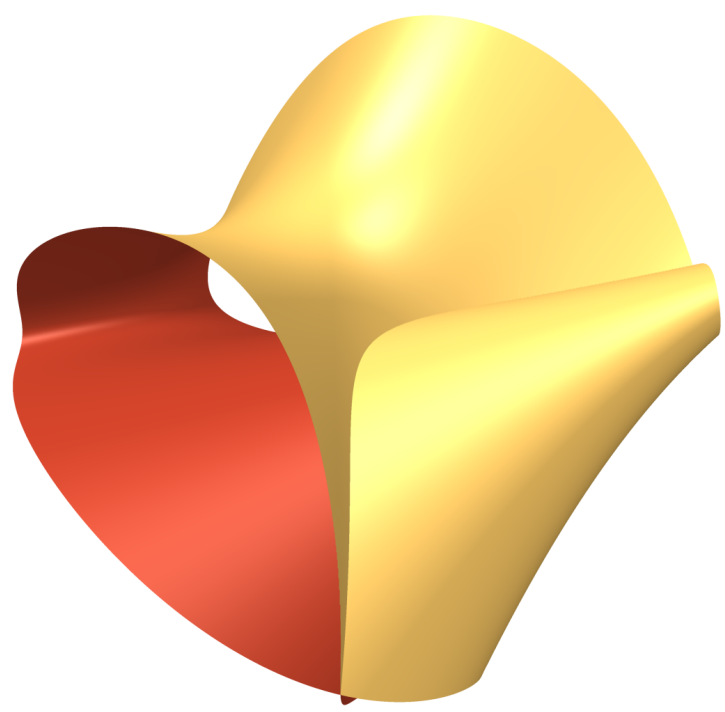

Figure 1: $-8 y^{4}+16 x y^{2} z+8 y^{3} z-8 x^{2} z^{2}-8 x y z^{2}-8 y^{2} z^{2}+2 x z^{3}+2 y z^{3}+4 z^{4}-$ $8 x y^{2}+2 y^{3}+8 x^{2} z+10 x y z-2 y^{2} z-2 x z^{2}-8 y z^{2}-6 x^{2}+2 x y+4 y^{2}=0$

\subsubsection{Hypersurfaces singular along a ruled cubic surface in $\mathbb{P}^{4}$}

A ruled cubic surface in $\mathbb{P}^{4}$ is the base locus of a net of quadrics of determinantal type (cf. Beauville [4, Prop. IV.7, p. 44)]; it's projectively equivalent to the subvariety $W$ defined by the ideal $\mathcal{I}_{W}$ of the $2 \times 2$ minors of the matrix $\left(\begin{array}{ccc}x_{0} & x_{1} & x_{2} \\ x_{2} & x_{3} & x_{4}\end{array}\right)$. It has Hilbert polynomial $P_{r c}(t):=(3 / 2) t^{2}+(5 / 2) t+1$. Denote by $\mathbb{W}_{r c}$ the corresponding component in Hilb $\mathbb{P}^{4}$. The family $\mathbb{W}_{r c}$ has dimension 18. The Hilbert polynomial of the subscheme $W_{r c}^{\prime}$ defined by $\mathcal{I}_{W}^{2}$ is $P_{W^{\prime}}(t)=(9 / 2) t^{2}-(5 / 2) t+2$. The family formed by subschemes of $\mathbb{P}^{4}$ defined by $\mathcal{I}_{W}^{2}$ for some $W \in \mathbb{W}_{r c}$ is not flat. The culprits are again in the orbit of the net $\mathbf{o}=\left\langle x_{0}^{2}, x_{0} x_{1}, x_{1}^{2}\right\rangle$, a legitimate member of $\mathbb{W}_{r c}$. Its square has Hilbert polynomial $5 t^{2}-5 t+5$ which is different from the expected. Blowing up as before produces a flat family $\mathbb{W}_{r c}^{\prime}$. Computational details are available in [28, Appendix G.1]. The polynomial that gives the 
degree of $\Sigma\left(\mathbb{W}_{r c}, d\right)$ is described below:

$$
\begin{gathered}
\operatorname{deg} \Sigma\left(\mathbb{W}_{r c}, d\right)=\frac{1089331}{2820745970948505600} d^{54}-\frac{4609327}{138135296519700480} d^{53} \\
+\frac{17053361977}{12432176686773043200} d^{52}-\frac{44006738257}{1243217668677304320} d^{51}+\frac{43540862009}{68559797904998400} d^{50} \\
-\frac{6776065867607}{822717574859980800} d^{49}+\frac{25203282464989}{329087029943992320} d^{48}-\frac{95461703632727}{205679393714995200} d^{47} \\
+\frac{3121945759267787}{3290870299439923200} d^{46}+\frac{13975371538743871}{987261089831976960} d^{45}-\frac{1762263793046822003}{9872610898319769600} d^{44} \\
+\frac{1571373547792223293}{1645435149719961600} d^{43}-\frac{18657333817850689}{21095322432307200} d^{42}-\frac{21162893089184824063}{822717574859980800} d^{41} \\
+\frac{8817237395388371983}{42070785078067200} d^{40}-\frac{7285835577039579827299}{7404458173739827200} d^{39}+\frac{18439965173115436460101}{2278294822689177600} d^{38} \\
\quad-\frac{30625726302752154570146789}{251751577907154124800} d^{37}+\frac{286671605346783151488709819}{201401262325723299840} d^{36} \\
-\frac{5957731889573498708183240461}{503503155814308249600} d^{35}+\frac{946219385360559194318492423}{13078004047124889600} d^{34} \\
-\frac{28843644632003758667785804741}{88853498084877926400} d^{33}+\frac{3586612308873070845414316631}{3702229086869913600} d^{32} \\
-\frac{2772990057804229211772760003}{3173339217317068800} d^{31}-\frac{173239617944054456458227898277}{17770699616975585280} d^{30} \\
+\frac{3107360934070968268891455300733}{44426749042438963200} d^{29}-\frac{1302777164405876523072798778669}{4936305449159884800} d^{28}
\end{gathered}
$$




$$
\begin{gathered}
+\frac{2175543494720246680252051667789}{3748506950455787520} d^{27}-\frac{15324266643945858395023213928441}{88853498084877926400} d^{26} \\
-\frac{583723723691983350730395768869707}{133280247127316889600} d^{25}+\frac{295008612506350533900867771909281}{14808916347479654400} d^{24} \\
-\frac{72882298518045984492971696381249}{1514548262810419200} d^{23}+\frac{3179423312365559691881647284007591}{59235665389918617600} d^{22} \\
+\frac{65074915758634148942372090942475703}{799681482763901337600} d^{21}-\frac{17650658027740832446748837419090939}{33566877054287216640} d^{20} \\
+\frac{43155219287681067897344483362302109}{35402565643193548800} d^{19}-\frac{30042531700267289895379997718912521}{22377918036191477760} d^{18} \\
-\frac{749894075579299475576086383836784223}{906305680465754849280} d^{17}+\frac{1152884114126290978903651885817821}{176296623184281600} d^{16} \\
-\frac{679247544279215190070362388445065693}{49980092672743833600} d^{15}+\frac{272444209645180356835326895182601977}{1851114543434956800} d^{14} \\
-\frac{14180655522525890878698424573977769}{8330015445457305600} d^{13}-\frac{17786673868531949329900173945074227}{694167953788108800} d^{12} \\
+\frac{8140256480874854682039834827204717}{148750275811737600} d^{11}-\frac{15847193428252892198587722393037621}{231389317929369600} d^{10} \\
+\frac{51203085967146132778275681925029671}{851933397830860800} d^{9}-\frac{415833099791358148948760413114949}{10846374277939200} d^{8} \\
-\frac{1981299728200259795937983}{242514155520} d^{3}+\frac{15743878343562160667}{7623616} d^{2} \\
+\frac{190922280640278098795730933090799}{10846374277939200} d^{7}-\frac{47833769039838754264953305641}{8608233553920} d^{6} \\
+\frac{2764737243980163013076109790463}{2560949482291200} d^{5}-\frac{1553358364438869321892260077}{17784371404800} d^{4}
\end{gathered}
$$

Note that the degree in $(28)$ is $54=(2+1) \times 18$, cf. $(2)$.

\subsubsection{Hypersurfaces Singular along a Segre 3-fold in $\mathbb{P}^{5}$}

The Segre variety $\mathbb{S}:=\mathbb{P}^{1} \times \mathbb{P}^{2} \subset \mathbb{P}^{5}$ has Hilbert polynomial $(1 / 2) t^{3}+$ $2 t^{2}+(5 / 2) t+1$. It moves in a family $\mathbb{W}_{\text {seg }}$ of dimension 24 . It is well known (cf. Harris [16, p. 99]) that the homogeneous ideal is spanned by a net of quadrics of determinantal type. Identifying $\mathbb{P}^{5}=\mathbb{P}\left(\operatorname{Hom}\left(\mathbb{C}^{2}, \mathbb{C}^{3}\right)\right)$, $\mathbb{S}$ corresponds to the locus of rank one matrices up to scalar. As in the previous 2 cases, the family formed by the subschemes of $\mathbb{P}^{5}$ defined by $\mathcal{I}_{W}^{2}$ for some $W \in \mathbb{W}_{\text {seg }}$ lacks flatness precisely along the nets coming from the Veronese-like embedding $\mathbb{G}\left(2, \mathcal{F}_{1}\right) \cong \mathbb{G}_{\mathbf{o}} \subset \mathbb{G}\left(3, \mathcal{F}_{2}\right),\left\langle L_{0}, L_{1}\right\rangle \mapsto$ 
$\left\langle L_{0}^{2}, L_{0} L_{1}, L_{1}^{2}\right\rangle$. Write $\widehat{\mathbb{X}}$ for the blowing up of $\mathbb{X}(=$ nets of quadrics of determinantal type) along $\mathbb{G}_{\mathbf{o}}$. It embeds in $\mathbb{X} \times \mathbb{G}\left(6, \mathcal{F}_{4}\right)$ and the exceptional divisor $\widehat{\mathbb{E}}$ affords the same description as in Proposition 14(iii). Scripts are available in [28, Appendix H].

Although we have all the information needed to calculate $\operatorname{deg} \Sigma\left(\mathbb{W}_{\text {seg }}, d\right)$ via Bott's residues formula, computations become prohibitive beyond $d=$ 28 , last entry in Table 1 . So we were not able to perform interpolation, which would require pushing $d$ up to $(3+1) \times 24$ (conjecturally).

\begin{tabular}{|c|c|}
\hline $\mathrm{d}$ & degree \\
\hline 4 & 4985292672535 \\
\hline 5 & 38085453623924002125608 \\
\hline 6 & 75285508677103874434199729447346 \\
\hline 7 & 6919928722801305898152558631141006297978 \\
\hline 8 & 42181954432466686484802366327946036350563667373 \\
\hline 9 & 30538531184782134440883223805188165885850765266730973 \\
\hline 10 & 4224340951726565859342587822879909669270072209918091111509 \\
\hline 11 & 158437528281133532734337703310993668084277908103801228619349318 \\
\hline 12 & 2080035353059957499641534559924163791462457116358313751435919907641 \\
\hline 13 & 11549735996636189943619254985547139290129087463355134074887299468381440 \\
\hline 14 & 31296770227603270473657644859463859788303319257226489697655766935282861144 \\
\hline 15 & 46218251138854455896028288030807107836206397262026919058025989004860345865068 \\
\hline 16 & 40573178025017053248163455791995253138333248830219749681901524680514920694647875 \\
\hline 17 & 22696403460389782282918120220096612693066990902486735463037695748458355012102065130 \\
\hline 18 & 8560094850432050145388608162764331545974912158826771912534187363304630242378140685505 \\
\hline 19 & 2280218446179281906894436399299532691147069188695294809825377606946754403932028306244123 \\
\hline 20 & 445913122370782785268625533245649250274532741301118606978525517483582671680154337345798650 \\
\hline 21 & 66136044830890785552763166513088475675562647217232322960605533153943919181299528743949231995 \\
\hline 22 & 7648060182749239379957328222725038044389468341441118678038359708033154622298562461760431031987 \\
\hline 23 & 706122123807470790783755440277242773510506336035275026531817959540511125994738199269690002855831 \\
\hline 24 & 53126049393404266440928946834127714486755547747259961078332514818104185788066175129628674092418346 \\
\hline 25 & 3315561352388199144671538442416320830215174679718026171794913021436371907104234446224006732128647329 \\
\hline 26 & 174334857471395667347731728239322112964231210603282356701591598514084204408291171080634141000772703155 \\
\hline 27 & 7829482987143513944990986949407455476367377747552625701320278751035638067417139596314040948040344857400 \\
\hline 28 & 303991364820542511002698414336553281396075120749252336213971319871871164262548779281153647072907136671375 \\
\hline
\end{tabular}

Table 1: $\operatorname{deg} \Sigma\left(\mathbb{W}_{\text {seg }}, d\right)$

\subsection{Surfaces singular along elliptic quartic curves}

An elliptic quartic curve in $\mathbb{P}^{3}$ is the complete intersection of a (unique) pencil of quadric surfaces. Avritzer \& Vainsencher [34], [3] obtained an explicit description of the component $\mathbb{W}_{e q c}$ of elliptic quartics of the Hilbert scheme $\operatorname{Hilb}_{4 t}\left(\mathbb{P}^{3}\right)$. This has been used in [9] for enumerating curves in cer- 
tain Calabi-Yau 3-folds, and in [6] for studying Noether-Lefschetz loci of systems of surfaces in $\mathbb{P}^{3}$. G. Gotzmann [13] has shown that $\mathrm{Hilb}_{4 t}\left(\mathbb{P}^{3}\right)$ consists of two irreducible components; the second one parameterizes unions of a plane quartic curve and a zero dimensional subcheme of $\mathbb{P}^{3}$ of length 2 .

Put $\mathbb{X}=\mathbb{G}\left(2, \mathcal{F}_{2}\right)$, the grassmannian of pencils of quadrics in $\mathbb{P}^{3}$. We summarize in the diagram below the construction of $\mathbb{W}_{\text {eqc }}$.

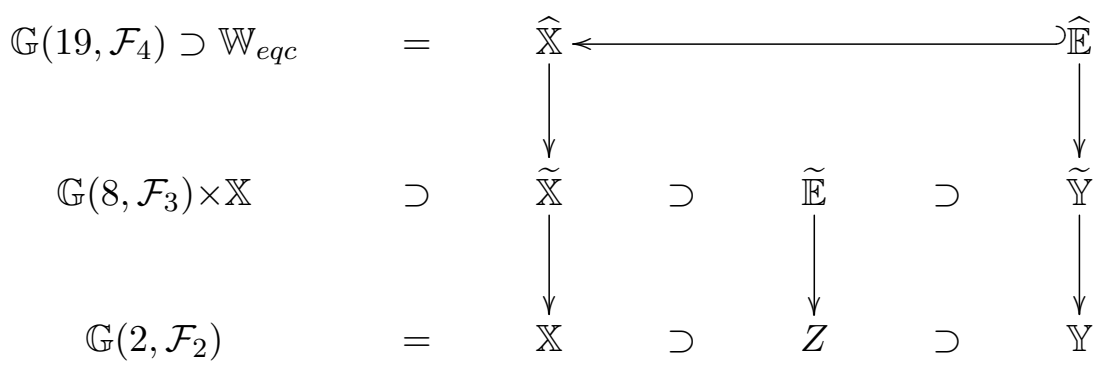

where
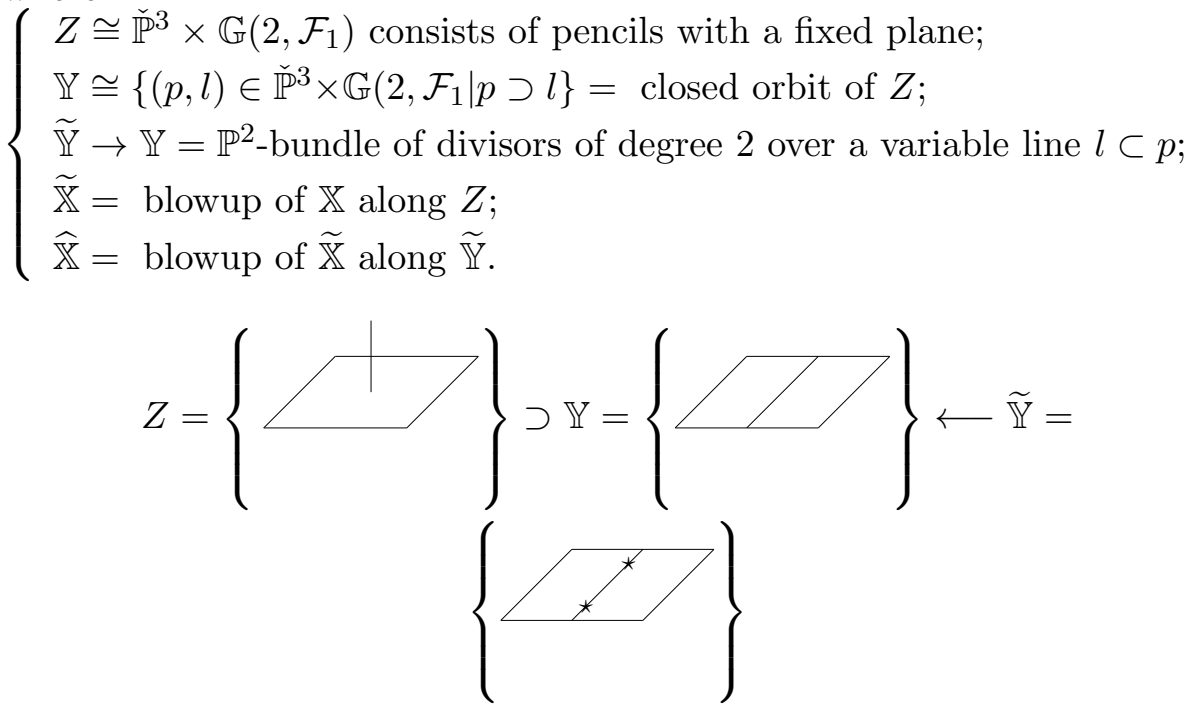

Let

$$
\mathcal{A} \subset \mathcal{F}_{2} \times \mathbb{X}
$$

be the tautological subbundle of rank 2 on our grassmannian of pencils of quadrics. There is a natural map of vector bundles on $\mathbb{X}$ induced by 
multiplication,

$$
\mu_{3}: \mathcal{A} \otimes \mathcal{F}_{1} \longrightarrow \mathcal{F}_{3} \times \mathbb{X}
$$

with generic rank 8 . The rank drops precisely over $Z$. Hence we have an induced rational map $\kappa: \mathbb{X} \rightarrow \mathbb{G}\left(8, \mathcal{F}_{3}\right)$. Blowing up $\mathbb{X}$ along $Z$, we find the closure $\widetilde{\mathbb{X}} \subset \mathbb{G}\left(8, \mathcal{F}_{3}\right) \times \mathbb{X}$ of the graph of $\kappa$. The fiber

$$
\widetilde{\mathbb{E}}_{(p, l)}=\mathbb{P}\left(\mathcal{F}_{3}^{l} / p \mathcal{F}_{2}^{l}\right)
$$

where $\mathcal{F}_{d}^{l}$ denotes the space of forms of degree $d$ vanishing on the line $l$. The fiber of $\widetilde{\mathbb{E}}$ over $\mathbf{y}:=\left(x_{0},\left\langle x_{0}, x_{1}\right\rangle\right) \in \mathbb{Y}$ contains the disjoint subspaces

$$
\mathbb{M}_{\mathbf{y}}:=\mathbb{P}\left(x_{1}^{2}\left(\mathcal{F}_{1} /\left\langle x_{0}\right\rangle\right)\right) \text { and } \mathbb{P}\left(\left(\mathcal{F}_{2} / \mathcal{F}_{2}^{l}\right)\right)=\widetilde{\mathbb{Y}}_{\mathbf{y}} .
$$

The latter embeds into $\widetilde{\mathbb{E}}_{(p, l)}$ via multiplication by $p:=x_{0}$ and coincides with the fiber of $\widetilde{Y}$. The former is the fiber of a $\mathbb{P}^{2}$-bundle

$$
\mathbb{M} \longrightarrow \mathbb{Y}
$$

to be further described in a moment.

Now, over $\widetilde{\mathbb{X}}$ we have a subbundle of cubic forms,

$$
\mathcal{B} \subset \mathcal{F}_{3} \times \widetilde{\mathbb{X}}
$$

of rank 8 obtained by pullback from the tautological subbundle over $\mathbb{G}\left(8, \mathcal{F}_{3}\right)$. Thus we get a map of multiplication

$$
\mu_{4}: \mathcal{B} \otimes \mathcal{F}_{1} \rightarrow \mathcal{F}_{4} \times \widetilde{\mathbb{X}}
$$

with generic rank 19. The scheme of zeros of $\bigwedge^{19} \mu_{4}$ is equal to $\widetilde{\mathbb{Y}}(29)$. In fact, it can be verified that each fiber of $\mathcal{B}$ is a linear system of cubics such that

- either it has a base locus equal to a curve with "correct" Hilbert polynomial $P_{\mathbb{W}_{e q c}}(t)=4 t$

- or it is of the form $p \cdot \mathcal{F}_{2}^{* *}$, meaning a linear system with fixed component a plane $p$, and $\mathcal{F}_{2}^{* *}$ denoting an 8-dimensional space of quadrics cutting a subscheme of $p$ of dimension 0 and degree 2 . 
The exceptional divisor $\widehat{\mathbb{E}}$ is the $\mathbb{P}^{8}$-bundle over $\widetilde{\mathbb{Y}}$ with fiber

$$
\begin{array}{r}
\widehat{\mathbb{E}}_{\left((p, l), y_{1}+y_{2}\right)}=\text { system of quartic curves in the plane } p \text { which } \\
\text { are singular at the "doublet" } y_{1}+y_{2} .
\end{array}
$$

Precisely, assuming the plane $p:=x_{0}$ and the line $l:=\left\langle x_{0}, x_{1}\right\rangle$, a typical doublet has homogeneous ideal of the form $\left\langle x_{0}, x_{1}, f\left(x_{2}, x_{3}\right)\right\rangle$, for some binary form $f, \operatorname{deg} f=2$. Our system of plane quartics lies in the ideal $\left\langle x_{1}, f\right\rangle^{2}=\left\langle x_{1}^{2}, x_{1} f, f^{2}\right\rangle$. Given a non-zero quartic $g$ in this ideal, we may form the ideal $J=\left\langle x_{0}^{2}, x_{0} x_{1}, x_{0} f, g\right\rangle,\left(e . g,\left\langle x_{0}^{2}, x_{0} x_{1}, x_{0} x_{2}^{2}, x_{2}^{4}\right\rangle\right)$. It can be checked that $J$ contains precisely 19 independent quartics and the Hilbert polynomial is correct. Moreover, the subscheme defined by $J^{2}$ has the expected Hilbert polynomial $12 t-16$. The preceding description suffices to get a hold on the fixed points on $\widehat{\mathbb{X}}(29)$ together with their tangent spaces as explained in Araújo [2] (after [9], [24]). However, as in the case of nets of quadrics, once we pass to the thickenings, one last blowup is required. The new center $\mathbb{M} \subset \widetilde{\mathbb{E}}$ is supported in the locus of $W \in \mathbb{W}_{\text {eqc }}$ where the subscheme of $\mathbb{P}^{3}$ defined by $\left(\mathcal{I}_{W}\right)^{2}$ has "wrong" Hilbert polynomial: flatness fails. In fact, points corresponding to an ideal like

$$
\left\langle x_{0}^{2}, x_{0} x_{1}, C\right\rangle \in \widetilde{\mathbb{E}}_{\left(x_{0},\left\langle x_{0}, x_{1}\right\rangle\right)},
$$

where $C$ denotes a cubic form arising from $x_{1}^{2} \cdot\left(\mathcal{F}_{1} /\left\langle x_{0}\right\rangle\right)$, are legitimate members of $\mathbb{W}_{e q c}$, whereas its square has a "bad" Hilbert polynomial (namely $13 t-20)$. Notation as in $(30),(31)$, let $\nu: \mathcal{A} \otimes \mathcal{B} \rightarrow \mathcal{F}_{5}$ be map of vector bundles over $\widetilde{\mathbb{X}}$ defined by multiplication. The generic rank of $\nu$ is 12 . Set

$$
\mathbb{M}=\text { scheme of zeros of } \bigwedge^{12} \nu
$$

In a way similar to Prop. 14, local calculations (cf. [28, Appendix I.1, p.177]) show that $\mathbb{M}$ is the indeterminacy locus of the natural rational map

$$
\widetilde{\mathbb{X}}-\rightarrow \mathbb{G}\left(12, \mathcal{F}_{5}\right)
$$

induced by $\nu$. One checks that $\mathbb{M}$ is the $\mathbb{P}^{2}$-bundle over $\mathbb{Y}$ which parameterizes the triples $\langle p, l, C\rangle$, where $p$ denotes a plane, $l=\left\langle p, p^{\prime}\right\rangle$ a 
line therein and $C$ a class in $\mathbb{P}\left(\left(p^{\prime}\right)^{2} \cdot \mathcal{F}_{1} /\langle p\rangle\right)$. We have the embedding $\mathbb{M} \subset \widetilde{\mathbb{E}}$ of bundles over $\mathbb{Y}$ such that in the fiber over any $(p, l) \in Y$ the point $\langle p, l, C\rangle$ with $C=\left(p^{\prime}\right)^{2} p^{\prime \prime} \bmod \langle p\rangle$ is mapped to the class $\overline{\left(p^{\prime}\right)^{2} \cdot p^{\prime \prime}} \in \mathbb{P}\left(\mathcal{F}_{3}^{l} / p \mathcal{F}_{2}^{l}\right)=\widetilde{\mathbb{E}}_{(p, l)}$. Consider the blow up diagram of $\widetilde{\mathbb{X}}$ along $\mathbb{M}$

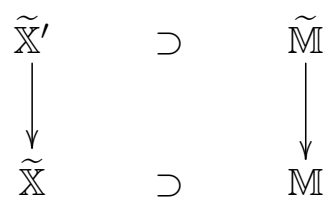

By construction $\widetilde{\mathbb{X}}^{\prime}$ embeds in $\widetilde{\mathbb{X}} \times \mathbb{G}\left(12, \mathcal{F}_{5}\right)$ as the closure of the graph of the rational map (33). Since $\mathbb{M}$ is disjoint from the blowup center $\widetilde{\mathbb{Y}}$ (cf. diagram 29), it follows that $\widetilde{\mathbb{Y}}$ lifts isomorphically to $\widetilde{\mathbb{Y}}^{\prime} \subset \widetilde{\mathbb{X}}^{\prime}$ so that the blowup of $\widetilde{\mathbb{X}}$ along $\widetilde{\mathbb{Y}}$ is naturally isomorphic to the blowup of $\widetilde{\mathbb{X}}^{\prime}$ along $\widetilde{\mathbb{Y}}^{\prime}$ over a neighborhood of $\widetilde{\mathbb{Y}}$. In special, only the fixed points of $\widehat{\mathbb{X}}$ over $\mathbb{M}$ are replaced by those in $\widetilde{\mathbb{M}}$. It turns out that a point like (32) is replaced by 9 fixed points in $\widetilde{\mathbb{M}}$ corresponding to ideals of the form

$$
\begin{gathered}
\left\langle x_{0}^{2}, x_{0} x_{1}, C\right\rangle^{2}+\langle m\rangle \\
m \in\left\{x_{0} x_{2} C, x_{0} x_{3} C, \frac{C^{2}}{x_{1}}, x_{0}^{2} x_{1} x_{2}^{2}, x_{0}^{2} x_{1} x_{2} x_{3}, x_{0}^{2} x_{1} x_{3}^{2}, x_{0}^{2} x_{0} x_{2}^{2}, x_{0}^{2} x_{0} x_{2} x_{3}, x_{0}^{2} x_{0} x_{3}^{2}\right\} .
\end{gathered}
$$

The technicalities of the final computation can be found in [28, Appendix I.1, p.177]. The polynomial that gives us the degree of $\Sigma\left(\mathbb{W}_{e q c}, d\right)$ is displayed below. Note once again that the degree is equal to $(1+1) \times$ $\operatorname{dim}\left(\mathbb{W}_{\text {eqc }}\right)=2 \times 16$, cp. $(2)$. 


$$
\begin{aligned}
& \operatorname{deg} \Sigma_{\mathbb{W}_{e q c}, d}=\frac{77991978249}{47023181004800} d^{32}-\frac{142130943}{922746880} d^{31}+\frac{8109239447979}{1175579525120} d^{30} \\
& -\frac{4150267051797}{20992491520} d^{29}+\frac{47676232841150619}{11755795251200} d^{28}-\frac{6615027446596551}{104962457600} d^{27} \\
& +\frac{128385059997089001}{167939932160} d^{26}-\frac{103459871906659801}{14129561600} d^{25}+\frac{893796960041917863271}{16277254963200} d^{24} \\
& -\frac{312845973151702414313}{1017328435200} d^{23}+\frac{4312587609200253695639}{4069313740800} d^{22} \\
& +\frac{6155781582234103357}{7266631680} d^{21}-\frac{1105621403101024328482787}{24415882444800} d^{20} \\
& +\frac{2134617904050477326290337}{5410337587200} d^{19}-\frac{1027704290752048951537337771}{476109707673600} d^{18} \\
& +\frac{1568309607110425883232529237}{223176425472000} d^{17}+\frac{399314335681097660200615893191}{57133164920832000} d^{16} \\
& -\frac{127911974311612787565094357769}{396758089728000} d^{15}+\frac{729760755266942589134714032019}{238054853836800} d^{14} \\
& -\frac{18285322486683264514566399967249}{892705701888000} d^{13}+\frac{15050777906503580350914982390277}{137339338752000} d^{12} \\
& -\frac{8362721204990643447960751421719}{17167417344000} d^{11}+\frac{178565283439979930078484872809}{98099527680} d^{10} \\
& -\frac{2731787128737717049736180171243}{476872704000} d^{9}+\frac{1125598445944774654288515801691861}{74392141824000} d^{8} \\
& -\frac{58025484355390407710374488759691}{1743565824000} d^{7}+\frac{16796039461040747482814365174429}{278970531840} d^{6} \\
& -\frac{8521350244073783951990040324653}{96864768000} d^{5}+\frac{599422208545470260381592707347}{5930496000} d^{4} \\
& -\frac{796327032680715287225577370219}{9081072000} d^{3}+\frac{434272227079029305979707333}{8072064} d^{2} \\
& -\frac{14906420412807524159489839}{720720} d+3713124778880030320 \text {. }
\end{aligned}
$$

Acknowledgement Thanks are due to Angelo F. Lopez for clarifying the argument on generic injectiveness (cf. Lemma9).

\section{References}

[1] A. B. Altman and S. L. Kleiman. Foundations of the theory of Fano schemes. Compositio Mathematica, 34(1):3-47, 1977. URL http:// www.numdam.org/item?id=CM_1977__34_1_3_0. 
[2] A. L. M. Araujo. Aplicações da Fórmula de Bott à Geometria Enumerativa. $\mathrm{PhD}$ thesis, Universidade Federal de Minas Gerais, 2009. URL http://www.mat.ufmg.br/intranet-atual/pgmat/ TesesDissertacoes/uploaded/Tese019.pdf.

[3] D. Avritzer and I. Vainsencher. The Hilbert scheme component of the intersection of two quadrics. Communications in Algebra, 27 (6):2995-3008, 1999. doi: 10.1080/00927879908826606. URL https: //doi.org/10.1080/00927879908826606.

[4] A. Beauville. Complex algebraic surfaces. London Mathematical Society student texts 34. Cambridge University Press, 2nd edition, 1996.

[5] D. F. Coray and I. Vainsencher. Enumerative formulae for ruled cubic surfaces and rational quintic curves. Commentarii Mathematici Helvetici, 61(1):501-518, Dec 1986. ISSN 1420-8946.

[6] F. Cukierman, A. Lopez, and I. Vainsencher. Enumeration of surfaces containing an elliptic quartic curve. Proceedings of the American Mathematical Society, 142(10):3305-3313, October 2014.

[7] W. Decker, G.-M. Greuel, G. Pfister, and H. Schönemann. Singular 4-1-1 - A computer algebra system for polynomial computations. http://www.singular.uni-kl.de, 2018.

[8] D. Eisenbud and J. Harris. The geometry of schemes, volume 197 of Graduate Texts in Mathematics. Springer-Verlag, New York, 2000. ISBN 0-387-98638-3; 0-387-98637-5.

[9] G. Ellingsrud and S. Strømme. Bott's formula and enumerative geometry. J. Amer. Math. Soc., 9(1):175-193, 1996.

[10] G. Ellingsrud, R. Piene, and S. A. Strømme. On the Variety of Nets of Quadrics Defining Twisted Cubic Curves, Space curves (Rocca Di Papa, 1985), volume 1266 of Lecture Notes in Mathematics, pages 84-96. Springer, 1987. 
[11] W. Fulton. Intersection Theory. Springer, 2nd edition, 1998.

[12] L. Göttsche. A conjectural generating function for numbers of curves on surfaces. Comm. Math. Phys., 196(3):523-533, 1998.

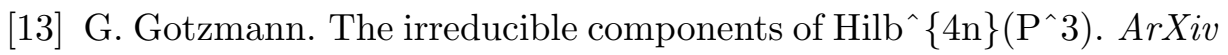
e-prints, Nov. 2008. URL https://arxiv.org/abs/0811.3160.

[14] D. Grayson and M. E. Stillman. Macaulay2 version 1.9.2, a software system for research in algebraic geometry. available at https://faculty.math.illinois.edu/Macaulay2/.

[15] A. Grothendieck. Techniques de construction et théorèmes d'existence en géométrie algébrique. IV. Les schémas de Hilbert. In Séminaire Bourbaki, Vol. 6, pages Exp. No. 221, 249-276. Soc. Math. France, Paris, 1995.

[16] J. Harris. Algebraic Geometry: A First Course. Number 133 in Graduate Texts in Mathematics. Springer-Verlag, 1992.

[17] R. Harris, J. Pandharipande. Severi degrees in cogenus 3. ArXiv e-prints, 1995. URL https://arxiv.org/abs/9504003v1.

[18] R. Hartshorne. Algebraic Geometry. Graduate Texts in Mathematics 52. Springer, 1977.

[19] H. Hironaka. Resolution of singularities of an algebraic variety over a field of characteristic zero. Annals of Math., 79:109-203, 1964.

[20] S. L. Kleiman. Intersection theory and enumerative geometry: a decade in review. In Algebraic geometry, Bowdoin, 1985 (Brunswick, Maine, 1985), volume 46 of Proc. Sympos. Pure Math., pages 321370. Amer. Math. Soc., Providence, RI, 1987. With the collaboration of Anders Thorup on $\S 3$.

[21] S. L. Kleiman and R. Piene. Node polynomials for families: methods and applications. Math. Nachr., 271:69-90, 2004. 
[22] Maple. Maplesoft, a division of Waterloo maple inc., Waterloo, Ontario. Version 2015.

[23] A. L. Meireles and I. Vainsencher. Equivariant intersection theory and Bott's residue formula - XVI Escola de álgebra - Part 1. Matemática Contemporânea, 20:1-70, 2001. URL http://mc.sbm.org.br/docs/ $\mathrm{mc} / \mathrm{pdf} / 20 / \mathrm{a} 1 . \mathrm{pdf}$.

[24] P. Meurer. The number of rational quartics on Calabi-Yau hypersurfaces in weighted projective space $\mathrm{P}\left(2,1^{4}\right)$. Mathematica Scandinavica, 78(1):63-83, 1996. URL http://www.jstor.org/stable/24492817.

[25] R. Piene and M. Schlessinger. On the Hilbert scheme compactification of the space of twisted cubics. American Journal of Mathematics, 107 (4):761-774, Aug.,1985. URL http://www.jstor.org/stable/2374355.

[26] M. Raynaud and L. Gruson. Critères de platitude et de projectivité. Techniques de "platification" d'un module. Invent. Math., 13:1-89, 1971.

[27] J. V. Rennemo. Universal polynomials for tautological integrals on Hilbert schemes. Geom. Topol., 21(1):253-314, 2017.

[28] W. D. Sellin. Enumeração de hipersuperfícies com subesquemas singulares. PhD thesis, Universidade Federal de Minas Gerais, 2018. URL https://arxiv.org/abs/1812.06129.

[29] E. Sernesi. Deformations of algebraic schemes, volume 334 of Grundlehren der Mathematischen Wissenschaften [Fundamental Principles of Mathematical Sciences]. Springer-Verlag, Berlin, 2006. ISBN 978-3-540-30608-5; 3-540-30608-0.

[30] Surfer2012. Mathematisches Forschungsinstitut Oberwolfach Visualization of algebraic surfaces. https:/github.com/Singular/ Sources/wiki/Installation-of-Surfer-on-Debian, 2012. 
[31] Y. Tzeng. Enumeration of singular varieties with tangency conditions. ArXiv e-prints, Mar. 2017. URL https://arxiv.org/abs/1703. $02513 \mathrm{v} 1$.

[32] I. Vainsencher. Hypersurfaces with up to six double points. Comm. Algebra, 31(8):4107-4129, 2003. Special issue in honor of Steven L. Kleiman.

[33] I. Vainsencher. Foliations singular along a curve. Trans. London Math. Soc, 2(1):80-92, July 2015. URL https://doi.org/10.1112/ tlms/tlv004.

[34] I. Vainsencher and D. Avritzer. Compactifying the space of elliptic quartic curves. Complex Projective Geometry(Trieste, 1989/Bergen, 1989), London Math. Soc. Lecture Note Ser., 179, Cambridge Univ. Press, Cambridge:47-58, 1992.

Weversson Dalmaso Sellin

Universidade Federal dos Vales do Jequitinhonha

Email: Mucuriweversson.sellin@ufvjm.edu.br

Israel Vainsencher

Universidade Federal de Minas Gerais

ivainsencher@ufmg.br 